\title{
SALT observations of the Be/X-ray binary A0538-66
}

\author{
A.F. Rajoelimanana* \\ Department of Physics, University of the Free State, PO Box 339, Bloemfontein 9300, SA \\ E-mail: rajoelimanana@ufs.ac.za
}

\section{P.J. Meintjes}

Department of Physics, University of the Free State, PO Box 339, Bloemfontein 9300, SA

E-mail: MeintjpJ@ufs.ac.za

\section{P.A. Charles}

School of Physics and Astronomy, Southampton University, Southampton SO17 1BJ, UK

E-mail: P.A.Charlesesoton.ac.uk

L.J. Townsend

ACGC, University of Cape Town, Private Bag X3, Rondebosch, 7701, SA

E-mail: townsendeast.uct.ac.za

The Be X-ray transient, A0538-66, is the most extreme LMC/SMC member of this class, exhibiting optical (and sometimes X-ray) outbursts with a period of 16.6d. More remarkably, long-term (MACHO) optical photometry revealed a large amplitude $(\sim 0.5 \mathrm{mag}) 421 \mathrm{~d}$ activity cycle which appears to control the appearance of the recurrent orbital outbursts/flares, as they are only seen during the minimum of the $421 \mathrm{~d}$ cycle. The nature of the physical link between these features is very poorly understood, but is likely related to properties of the Be star's equatorial disc. We will present our recent results on the long-term spectroscopic observations of this LMC BeX system using both broad-band (wide wavelength coverage) as well as high resolution optical spectra obtained from the Southern African Large Telescope (SALT). These observations enable us to probe both the evolution of the Be star envelope and the details of its interaction with the neutron star in its presumed highly eccentric $(\mathrm{e} \sim 0.7$ ) orbit. The high resolution spectra also allows us to derive its rotational velocity and spectral classification.

SALT Science Conference 2015 -SSC2015-

1-5 June, 2015

Stellenbosch Institute of Advanced Study, South Africa

\footnotetext{
*Speaker.
} 


\section{Introduction}

The Magellanic Clouds are known to host a large number of high-mass X-ray binaries (HMXBs) when compared to our Galaxy. The majority of these HMXBs are Be/X-ray binaries (BeX) in which a neutron star orbits around a massive $\left(\geq 10 \mathrm{M}_{\odot}\right)$ and rapidly rotating $(\geq 80 \%$ of break-up velocity) Be star [1]. The orbit of the system is generally very wide ( $P 20-200 \mathrm{~d}$ ) and eccentric $(0.1<\mathrm{e}<0.9)$. The neutron star (usually an X-ray pulsar) accretes mass from the equatorial disc around the $\mathrm{Be}$ star at or near periastron passage, giving rise to periodic outbursts that may be observed over a wide range of wavelengths (e.g. X-ray, optical, IR). Be stars are characterized by emission spectral lines and an excess of infrared emission originated from an extended circumstellar disc surrounding the B star.

The recurrent X-ray transient A0538-66 was discovered in 1977 when two major X-ray outbursts, separated by $\sim 17 \mathrm{~d}$, were observed with the Ariel $V$ satellite [2]. Subsequently, several outbursts from the source were observed with the HEAO-1 modulator collimator which was found to have a periodicity of $16.66 \mathrm{~d}[3,4,5]$. X-ray pulsations of $69 \mathrm{~ms}$ were detected in one of the strong X-ray outbursts observed with the Einstein (HEAO-2) Observatory [6], which implies that the compact object is a rapidly spinning neutron star. The optical counterpart was identified as a bright ( $\mathrm{B} \sim 15 \mathrm{mag}$ ) Be star, and optical spectroscopy suggests it to be a member of Large Magellanic Cloud (LMC) $[4,7]$. This distance implies that the observed peak X-ray luminosity in the first year of detection was very large $\left(\sim 10^{39} \mathrm{erg} \mathrm{s}^{-1}\right)$, substantially super-Eddington for a $1.4 \mathrm{M}_{\odot}$ neutron star, and still the highest in its class.

Archival plates photometry taken with the UK Schmidt telescope shows that the 15th mag B star counterpart has brightened by 2 mag in optical during the X-ray outbursts [5]. An orbital period of $16.6515 \mathrm{~d}$ was derived. This was confirmed by [8], who observed photometrically four consecutive outbursts of late 1981 and reported that the source can reach $12^{\text {th }}$ mag in optical. Moreover, this large optical brightening was accompanied by the sudden appearance of He II $\lambda 4686$ emission lines in their spectra. However, since late 1983, none of these huge outbursts have been observed, but much weaker outbursts (few tenth of a magnitude) were seen in both optical and X-ray observations. Based on optical and UV spectroscopy, the optical counterpart of A0538-66 was classified by [9] as a B2 III-V star. However, [10] suggests an earlier spectral type of B1 based on spectroscopic data taken during quiescence.

\section{SALT optical spectroscopy and data reduction}

The spectroscopic observations for A0538-66 were obtained with the Robert Stobie Spectrograph (RSS) [11, 12] on the Southern African Large Telescope (SALT) [13, 14] at SAAO, Sutherland. The data was taken between the 2011-3 and 2012-2 observing semesters. For each observing night we obtained both a broad-band and high resolution spectrum.

(1) Broad-band spectra: The PG0900 grating, with a slit width of 1.0", was used at two different angles $\left(12.5^{\circ}\right.$ and $\left.20.0^{\circ}\right)$ to provide an wavelength range of $\sim 3200-9000 \AA$, a dispersion of $1.0 \AA \mathrm{pixel}^{-1}$ and spectral resolution of $\sim 4.20 \AA$.

(2) High resolution spectra: The PG3000 grating was used at an angle of 40.5 with a slit width of 0.6 ". This gives a dispersion of $0.23 \AA$ pixel $^{-1}$ and spectral resolution of $0.7 \AA$. 
The data from each $2048 \times 4096$ CCD detector were pre-reduced with the IRAF package PYSALT [15] which include overscan, gain and cross-talk correction. The cosmic ray events were cleaned using the routine L.A.COSMIC [16]. Wavelength calibration was performed using the standard IRAF software tasks in TWODSPEC (identify, reidentify, fitcoords and transform). The spectra were flux calibrated using the sensitivity curve derived from the observed spectral energy distribution of spectrophotometric standard stars. One-dimensional spectra were then extracted using the IRAF task APALL.

\section{Broad-band spectra}

The two sets of spectra obtained from the two different settings of PG0900 were combined to give a wide wavelength coverage $(\sim 3200-9000 \AA)$. The spectra taken on the night of 2012 Oct 27 was used as its $\mathrm{H} \alpha$ line is the least affected by emissions from the equatorial disc. We then de-reddened the broad-band spectra using an extinction of $\mathrm{E}(\mathrm{B}-\mathrm{V})=0.11$ and compare it to synthetic spectra convolved with Gaussian with FWHM $=4.2 \AA$ to match the resolution of our spectra. We used the BSTAR2006 grids of a model spectrum from [17] and assuming a model atmosphere with half-solar chemical composition $\left(\mathrm{Z}=\mathrm{Z}_{\odot} / 2\right.$, LMC metallicity) and microturbulent velocity of $\xi=2 \mathrm{~km} \mathrm{~s}^{-1}$. The model spectra were also broadened to $v \sin i=200 \mathrm{~km} \mathrm{~s}^{-1}$ (see. Section 6 for derivation of $v \sin i$ ). The best fit model parameter values estimated from minimizing the $\chi^{2}$ corresponds to $T_{\text {eff }}=25000 \mathrm{~K}$ and $\log g=3.5$. Figure 1 shows the observed combined spectrum taken on the night of 2012-Oct-27 compared to the best fit model.

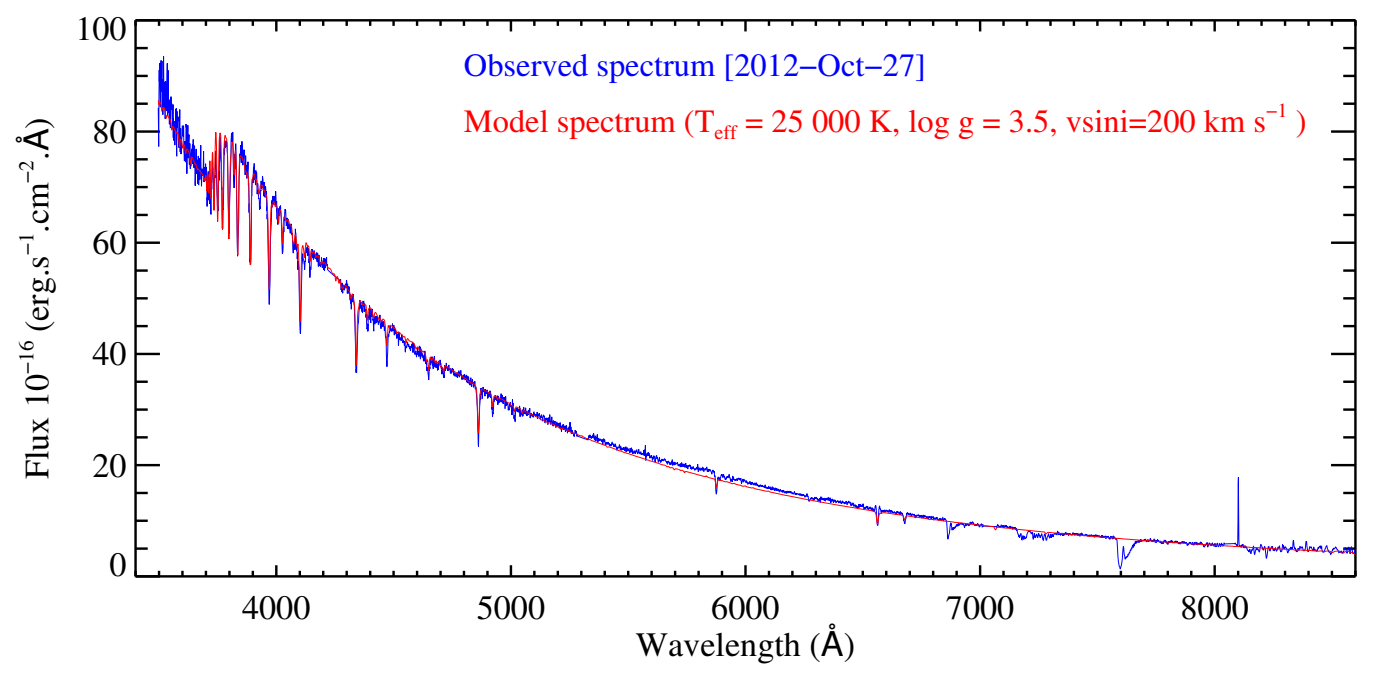

Figure 1: Broad-band spectrum of A0538-66 taken near the optical maxima (20121027) compared to the best fit model with $v \sin i=200 \mathrm{~km} \mathrm{~s}^{-1}, T_{\text {eff }}=25000 \mathrm{~K}$ and $\log g=3.5$.

\section{4. $H \alpha$ equivalent width and $V / R$ variations}

The evolution $\mathrm{H} \alpha$ line profile of A0538-66 is represented in Figure 2 which shows clearly a variability both in shape and strength. It exhibits a wide range of profiles such as single-peaked 
(2011-Oct-07), double-peaked (e.g. 2013-Jan-01), pure absorption (e.g. 2012-Oct-27) and shell profile (e.g. 2012-Aug-30). Furthermore, the long-term changes in the relative strength of the violet (V) and red (R) components of the observed $\mathrm{H} \alpha$ emission profiles, known as the "V/R variations", are clearly seen.
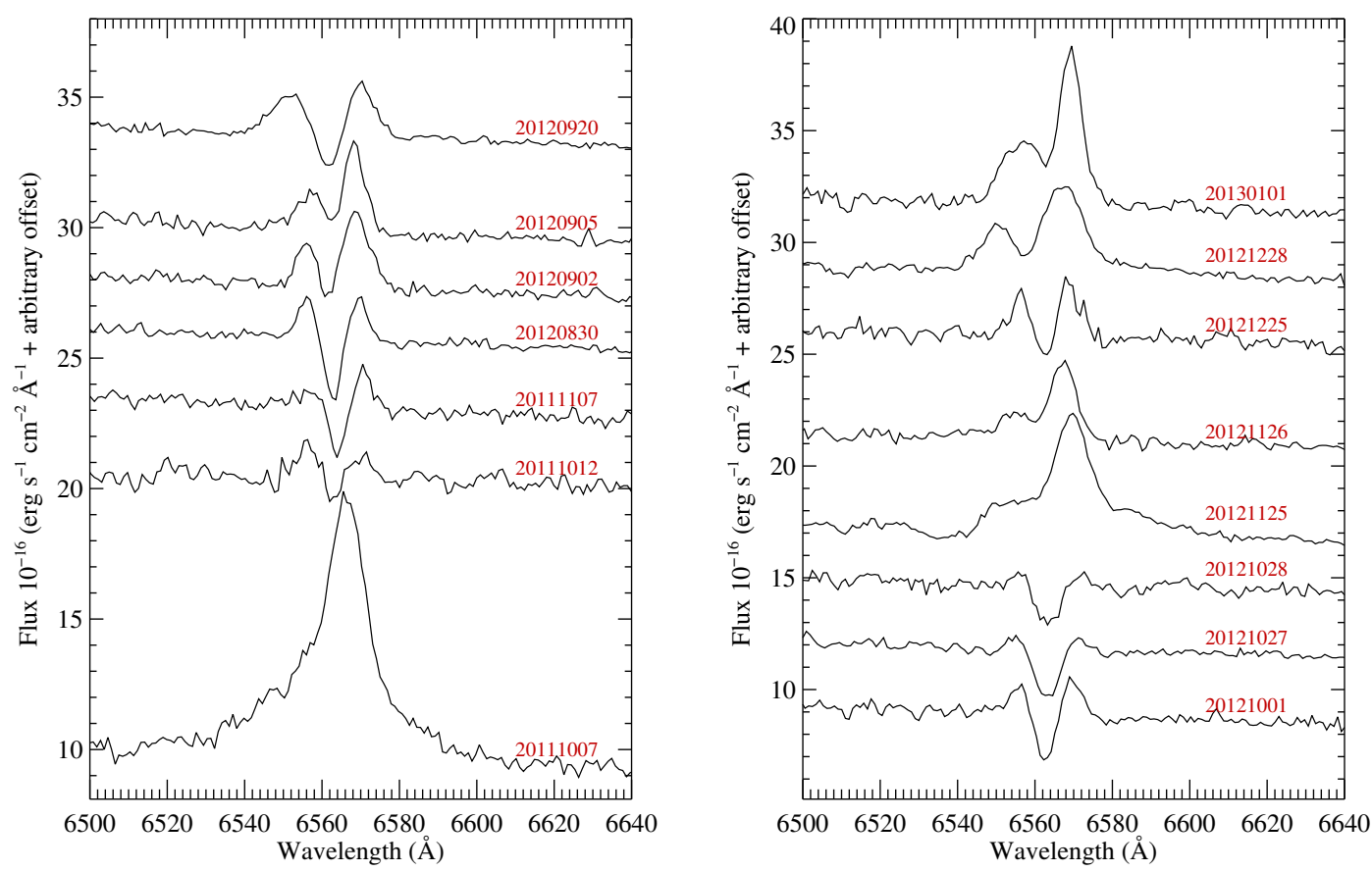

Figure 2: SALT/RSS spectra of the Be/X-ray system A0538-66 showing the evolution of its $\mathrm{H} \alpha$ emission line profiles.

We measured the line parameters by fitting the profiles with one, two or three Gaussians depending on whether the $\mathrm{H} \alpha$ line exhibits single, double-peaked or shell profiles. As the central absorption line goes beyond the continuum for shell profile, a third Gaussian component (in absorption) is needed to obtain a good fit.

The evolution of the equivalent width as well as the logarithm of the ratio V/R derived from the fits are shown in Figure 3 (left). The $\mathrm{H} \alpha$ equivalent width and V/R ratio phased with the orbital period of $16.6409 \mathrm{~d}$ and ephemeris derived by [18] are shown in Figure 3 (right). During the periods outside the periastron passage, the $\mathrm{H} \alpha$ equivalent width was well stable at about $-2 \AA$ and the V/R ratio was about -0.05 . This means that outside periastron the Be equatorial disc was not affected by the approaching neutron star. However, near periastron the equivalent width is increasing and peaking around $0.64 \mathrm{~d}$ after periastron with a maximum $\operatorname{EW}(\mathrm{H} \alpha)=20.9 \AA$. The V/R ratio also shows an excess emission on the red side of the $\mathrm{H} \alpha$ profile around periastron. This is interpreted as related to the variation of the size and mass of the Be equatorial disc, caused by its interaction with the neutron star near periastron passage.

\section{Spectral classification}

For spectral classification we used the temperature criteria suggested by [19] and [20] which 

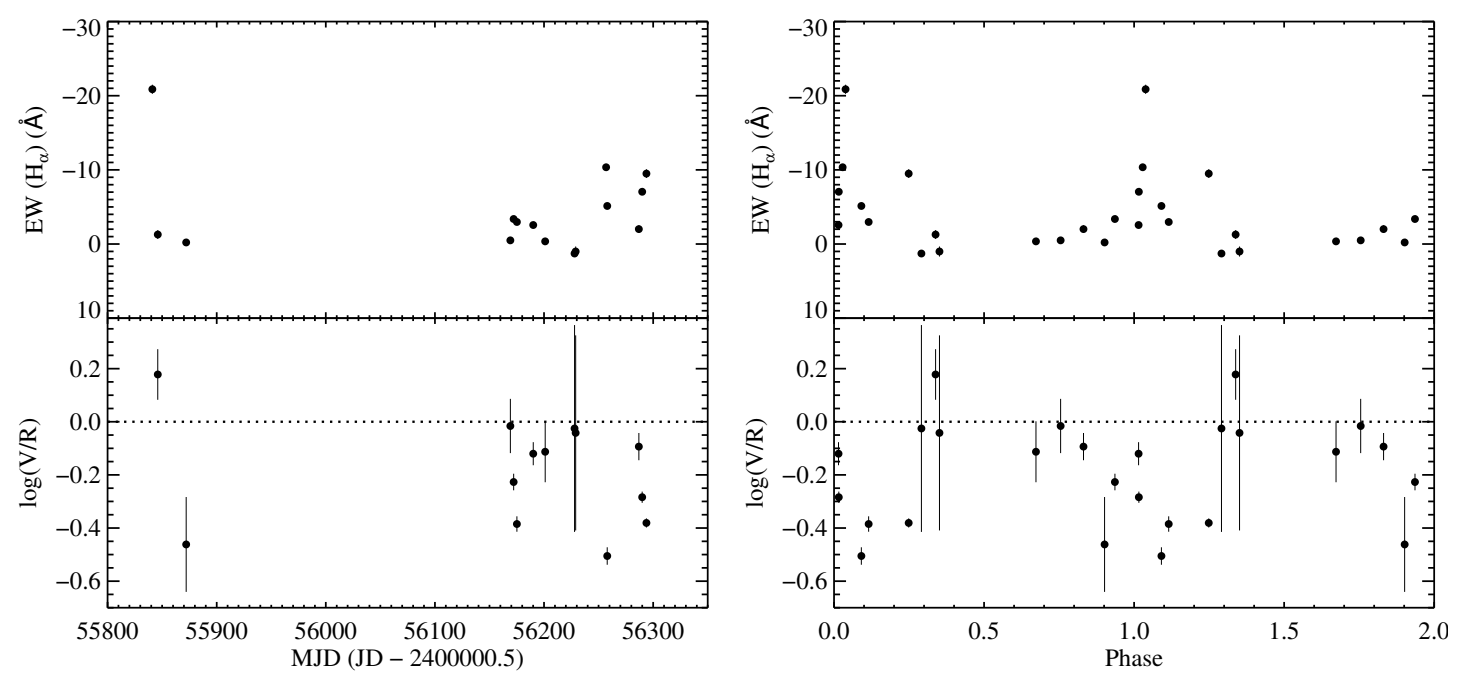

Figure 3: Left: Evolution of the $\mathrm{H} \alpha$ equivalent width (top) and V/R ratio (bottom). Right: $\mathrm{H} \alpha$ equivalent width and V/R ratio folded on the $16.6409 \mathrm{~d}$ period.

is based on the presence of the HeII $(\lambda 4200, \lambda 4541, \lambda 4686)$ lines for the earliest subtypes and on the metal line strengths for the latest subtype. The average high resolution blue spectrum (4000 - $4700 \AA$ ) of A0538-66 obtained with the SALT/RSS is shown in Figure 4 on which all identified main spectral features are marked and labeled. The spectra are mostly dominated by Balmer and neutral helium (He I) lines which is typical for an early-type B star. The absence of the ionized helium line at $4686 \AA$ indicates a spectral type later than B0.5. The relative weakness of the Mg II 4481 line suggests a spectral type earlier than B2. The Si III triplet at $\lambda 4552, \lambda 4568$ and $\lambda 4575$ are clearly visible which suggests a spectral type of B1-B1.5. The detection of weak Si IV lines ( $\lambda 4088, \lambda 4116)$ and the strength of the absorption lines of the C III + O II blends at $4070 \AA$ and $4650 \AA$ indicates a B1 spectral-type source.

The strength of the metal lines is a good indicator of luminosity class for B stars. The ratio between the Si III 4552 and the He I 4387, which is the primary luminosity criterion for B1 subtype, suggests an evolved star. The strength of the OII lines at $\lambda 4070$ (blend), $\lambda 4415$ and $\lambda 4640$ (blend), which is rising with increasing luminosity, also indicates a luminosity class of III. We conclude, therefore, that the optical counterpart of A0538-66 is a B1e III star which is a slightly earlier spectral type than the previously reported (B2 III-V). This spectral type is in good agreement with the stellar parameter $\left(T_{\text {eff }}=25000 \mathrm{~K}\right.$ and $\log g=3.5$ ) derived above from the fitting of the broad-band spectra.

\section{Rotational velocity}

Be stars are believed to rotate close to the break-up velocity. To estimate the projected rotational velocity, we used the relations between the rotational velocity and the full width half maximum (FWHM) of He I lines given in [21]. We measured the FWHMs of four He I lines ( $\lambda 4026$, $\lambda 4143, \lambda 4387, \lambda 4471$ ) from the $30^{\text {th }}$ August 2012 spectrum, which provides the highest $\mathrm{S} / \mathrm{N}$, and 


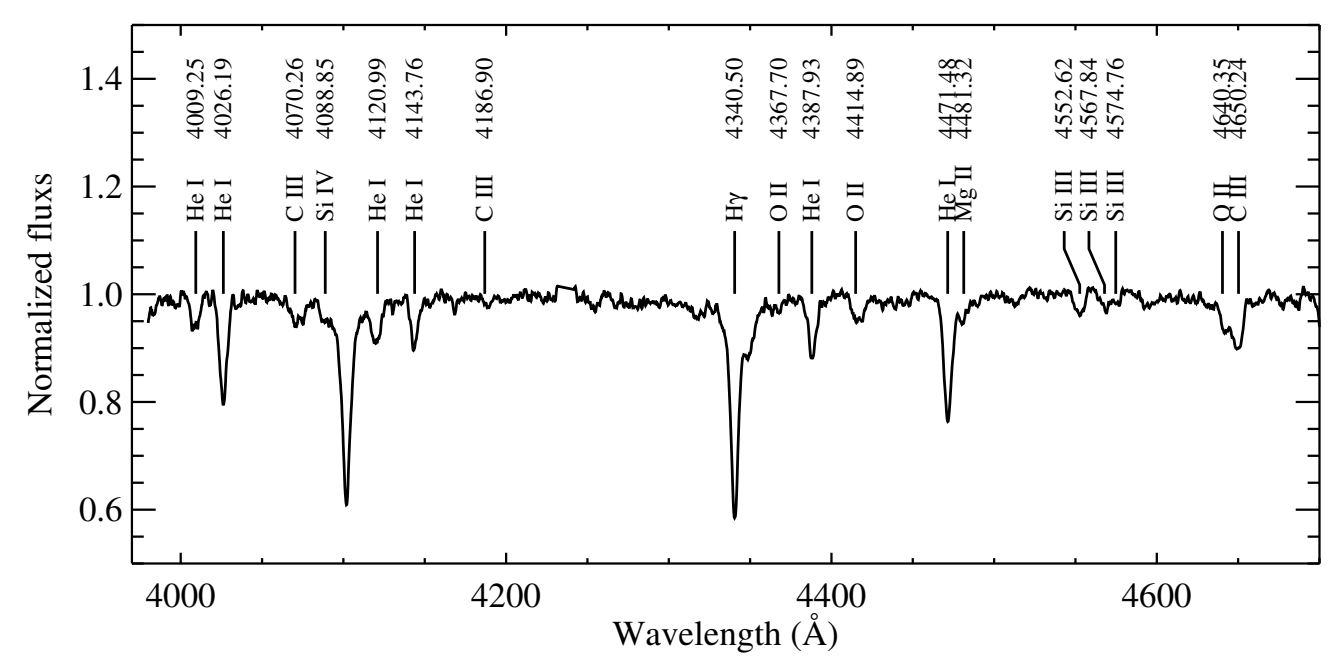

Figure 4: High resolution spectrum of A0538-66 in which all identified lines are marked.

translated them to a rotational velocity. The instrumental broadening was corrected by subtracting in quadrature the instrumental resolution of our spectra $\left(50 \mathrm{~km} \mathrm{~s}^{-1}\right)$ from the measured values of $v \sin i$. We found a weighted average of $v \sin i=198 \pm 21 \mathrm{~km} \mathrm{~s}^{-1}$.

We can also estimate the projected rotational velocity by comparing the observed spectra with a grid of synthetic spectra broadened from $50-500 \mathrm{~km} \mathrm{~s}^{-1}$ in steps of $10 \mathrm{~km} \mathrm{~s}^{-1}$, using the program rotin3. The model spectra were convolved with Gaussian with FWHM $=0.7 \AA$, in order to match the resolution of our observed spectra. We used the same model as above but assuming $T_{\text {eff }}=25000 \mathrm{~K}, \log g=3.5, \mathrm{Z}=\mathrm{Z}_{\odot} / 2$ and $\xi=2 \mathrm{~km} \mathrm{~s}^{-1}$. The projected rotational velocity which minimizes the $\chi^{2}$ corresponds to $v \sin i=200 \mathrm{~km} \mathrm{~s}^{-1}$ which is in good agreement with the previous value.

\section{Conclusions}

We have presented the results of long-term SALT spectroscopic observations of the optical counterpart to A0538-66. We found a clear variation both in strength and shape of the $\mathrm{H} \alpha$ line profiles in our spectra which implies that the structure of the equatorial disk around the Be star is changing over the years. This must be associated with the formation and dissipation of the equatorial disc around the B star as discussed in [22, 23, 24]. The ratio between the peak of the violet $(\mathrm{V})$ and red $(\mathrm{R})$ component of the $\mathrm{H} \alpha$ profile is also changing during the observations, indicating that the Be equatorial disc is slightly distorted.

From the broad-band spectra, we were able to estimate the stellar atmospheric parameter $\left(T_{\text {eff }}\right.$ $=25000 \mathrm{~K}, \log g=3.5$ ). We refined the spectral classification of its optical counterpart to B1e III which is earlier than the spectral type found in the literature, namely B2 III-V, but in good agreement with the stellar parameter derived above. The width of the He I lines in our high resolution spectra suggests a rotational velocity of $v \sin i=198 \pm 21 \mathrm{~km} \mathrm{~s}^{-1}$. 


\section{Acknowledgments}

All of the observations reported in this paper were obtained with the Southern African Large Telescope (SALT) under programs 2011-3-RSA_UKSC-008 (PI: Charles), 2012-1-RSA_UKSC005 and 2012-2-RSA_UKSC-003 (PI: Rajoelimanana)

\section{References}

[1] P. Reig, Be/X-ray binaries, Ap\&SS 332 (2011) 1-29.

[2] N. E. White and G. F. Carpenter, The recurrent X-ray transient A0538-66, MNRAS 183 (1978) 11P-15P.

[3] M. D. Johnston, H. V. Bradt, R. E. Doxsey, R. E. Griffiths, D. A. Schwartz, and J. Schwarz, Location of the recurrent /LMC/X-ray transient A0538-66 with the HEAO 1 scanning modulation collimator, ApJ 230 (1979) L11-L14.

[4] M. D. Johnston, R. E. Griffiths, and M. J. Ward, Improved position and new optical candidate for A0538-66, Natur 285 (1980) 26.

[5] G. K. Skinner, Observations of optical flares in the recurrent X-ray transient A0538-66, Natur 288 (1980) 141-143.

[6] G. K. Skinner, D. K. Bedford, R. F. Elsner, D. Leahy, M. C. Weisskopf, and J. Grindlay, Discovery of 69 MS periodic X-ray pulsations in A0538-66, Natur 297 (1982) 568-570.

[7] M. Pakull and A. Parmar, The optical counterpart of A 0538-66, A\&A 102 (1981) L1-L4.

[8] R. H. Densham, P. A. Charles, J. W. Menzies, M. van der Klis, and J. van Paradijs, Four outburst cycles of A0538-66 - Evidence for a rapidly evolving envelope around the primary, MNRAS 205 (1983) 1117-1133.

[9] P. A. Charles, L. Booth, R. H. Densham, and 6 colleagues, Extreme variability in the Be-type, periodic recurrent X-ray transient A0538-66 - A highly eccentric interacting binary, MNRAS 202 (1983) 657-682.

[10] J. B. Hutchings, D. Crampton, A. P. Cowley, E. Olszewski, I. B. Thompson, and N. Suntzeff, The optical orbit of the X-ray pulsar binary 0535 - 668 (= A0538 - 66), PASP 97 (1985) 418-422.

[11] E. B. Burgh, K. H. Nordsieck, H. A. Kobulnicky, T. B. Williams, D. O’Donoghue, M. P. Smith, and J. W. Percival, Prime Focus Imaging Spectrograph for the Southern African Large Telescope: optical design, SPIE 4841 (2003) 1463-1471.

[12] H. A. Kobulnicky, K. H. Nordsieck, E. B. Burgh, M. P. Smith, J. W. Percival, T. B. Williams, and D. O'Donoghue, Prime focus imaging spectrograph for the Southern African large telescope: operational modes, SPIE 4841 (2003) 1634-1644.

[13] D. A. H. Buckley, G. P. Swart, and J. G. Meiring, Completion and commissioning of the Southern African Large Telescope, SPIE 6267 (2006) 62670Z.

[14] D. O'Donoghue, D. A. H. Buckley, L. A. Balona, and 48 colleagues, First science with the Southern African Large Telescope: peering at the accreting polar caps of the eclipsing polar SDSS J015543.40+002807.2, MNRAS 372 (2006) 151-162.

[15] S. M. Crawford, M. Still, P. Schellart, and 14 colleagues, PySALT: the SALT science pipeline, SPIE 7737 (2010) 773725. 
[16] P. G. van Dokkum, Cosmic-Ray Rejection by Laplacian Edge Detection, PASP 113 (2001) 1420-1427.

[17] T. Lanz and I. Hubeny, A Grid of NLTE Line-blanketed Model Atmospheres of Early B-Type Stars, ApJS 169 (2007) 83-104.

[18] A. F. Rajoelimanana, P. A. Charles, P., J. Meintjes, L. J. Townsend, and A. Udalski, MNRAS, in preparation (2016)

[19] C. J. Evans, I. D. Howarth, M. J. Irwin, A. W. Burnley, and T. J. Harries, A $2 d F$ survey of the Small Magellanic Cloud, MNRAS 353 (2004) 601-623.

[20] V. Antoniou, D. Hatzidimitriou, A. Zezas, and P. Reig, Optical Spectroscopy of 20 Be/X-ray Binaries in the Small Magellanic Cloud, ApJ 707 (2009) 1080-1097.

[21] I. A. Steele, I. Negueruela, and J. S. Clark, A representative sample of Be stars . I. Sample selection, spectral classification and rotational velocities, A\&AS 137 (1999) 147-156.

[22] C. Alcock, R. A. Allsman, D. R. Alves, and 23 colleagues, A 421-d activity cycle in the BeX recurrent transient A0538-66 from MACHO monitoring, MNRAS 321 (2001) 678-684.

[23] K. E. McGowan and P. A. Charles, On the stability of the 421-d periodicity in A0538-66, MNRAS 339 (2003) 748-756.

[24] A. F. Rajoelimanana, P. A. Charles, and A. Udalski, Very long-term optical variability of high-mass X-ray binaries in the Small Magellanic Cloud, MNRAS 413 (2011) 1600-1622. 